\title{
REFLECTIVITY STUDIES OF PASSIVE MICROWAVE CALIBRATION TARGETS AND ABSORPTIVE MATERIALS*
}

\author{
Dazhen Gu, Amanda E. Cox, Derek Houtz, David K. Walker, James Randa, and Robert L. Billinger
}

National Institute of Standards and Technology, Boulder, CO

\begin{abstract}
We report on the characterization of blackbody reflections as a part of the recent progress on the development of brightness standards for microwave remote sensing at National Institute of Standards and Technology (NIST). Three blackbody targets at variable temperatures used for airborne and/or satellite systems along with an aluminum plate were measured in terms of their reflection coefficients by horn antennas in connection with a vector network analyzer (VNA) in the WR-42 waveguide band. Precision measurements of reflection are needed for blackbody emissivity computation to check against the brightness temperature measurement of blackbody targets. All experiments were conducted in two distance ranges by free-space methods in an anechoic chamber. Preliminary results show negligible reflections from the calibration targets, indicating near ideal blackbody characteristics in the measured frequency range.
\end{abstract}

Index Terms - Blackbody targets, brightness standard, free-space measurement, reflection coefficients

\section{INTRODUCTION}

After an interlude of several years, NIST has recently resumed efforts to develop a primary standard for microwave brightness temperature. The previous work is summarized in [1]. Currently, the principal goal [2] is to develop a standard radiometer, traceable to fundamental primary noise standards, but we are also taking steps toward characterizing standard targets. An early step in the characterization of calibration targets is to measure their reflectivity, and this paper reports initial measurement results for the monostatic reflection coefficient in normal incidence.
Such measurements constitute a first step toward measurement of the emissivity of the targets, which also requires full angular information about the scattered and reradiated power (as in [3]). The reflection coefficient at normal incidence is also of interest in its own right, since reflections from the target can have a significant effect on the noise figure of the radiometer, if it is not isolated [4].

\section{MEASURMENT SETUP}

We measured the reflection coefficient from three different calibration targets at $0.5 \mathrm{GHz}$ increments in the range from $18 \mathrm{GHz}$ to $26.5 \mathrm{GHz}$. In addition to the calibration targets, we also measured the reflection coefficient from a flat metal plate, for comparison. Measurements were made for two different ranges of separation distances between antenna and target. The measurements used a pyramidal standard-gain horn connected to a commercial vector network analyzer (VNA). The VNA was calibrated at the input flange to the antenna, and all measured reflection coefficients are with respect to this reference plane. The measurements were performed in the NIST anechoic chamber, which has been well-characterized for use in the $400 \mathrm{MHz}$ to $40 \mathrm{GHz}$ frequency range [5]. The targets were mounted on a precise positioning system that operates over a longitudinal range of approximately $4 \mathrm{~m}$. The rails and all metallic parts of the cart are covered with rf absorber. The minimum separation distance between antenna and target was dictated by the minimum cart location and the support structure for the target. For the calibration targets, this minimum distance was $36.5 \mathrm{~cm}$; for the metal plate, it was $43.7 \mathrm{~cm}$, representing the offset distances as opposed to the cart position. The cart position was then scanned from $0 \mathrm{~cm}$ to $10 \mathrm{~cm}$ and

\footnotetext{
${ }^{*}$ U. S. government work, not subject to U.S. copyright
} 


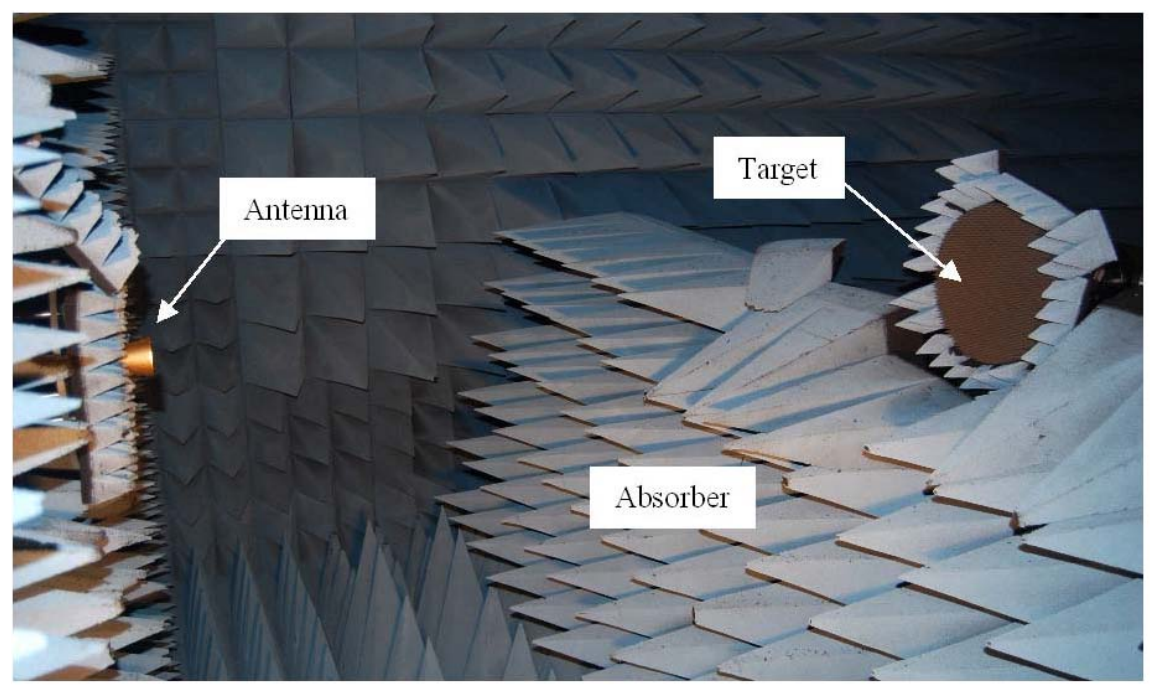

Figure 1. Measurement setup in anechoic chamber

from $225 \mathrm{~cm}$ to $235 \mathrm{~cm}$. A photo of the antenna and cart, with one of the targets mounted on the cart, is shown in Figure 1. The three calibration targets were circular discs, two of radius $18.034 \mathrm{~cm}(7.1 \mathrm{in})$, and one of radius $45.72 \mathrm{~cm}$ (18 in), all borrowed from a group at NASA Goddard Space Flight Center. Each target consisted of an iron-loaded epoxy coating on a machined aluminum tetrahedral pyramidal substrate. The two smaller targets differed from each other in the length (and aspect ratio) of the pyramids. Most measurements were performed with the calibration targets at ambient temperature (nominally $296 \mathrm{~K}$ ), but for some measurements a heating element was affixed to the back of the target, and the target temperature was raised to approximately $350 \mathrm{~K}$, as measured by platinum resistance thermometers (prt) embedded in the target. (The temperature varied somewhat, depending on prt, target, and time, but it was in the range from $349.7 \pm 0.7 \mathrm{~K}$ )

The calibration target is not the only source of reflections in our measurement setup. Although the anechoic chamber and the cart are designed to minimize any extraneous reflections, they are not perfect, and there are some small reflections from the cart and the chamber walls. More importantly, the standard-gain antenna is itself a source of reflections, from the junction where waveguide meets flare and from the aperture plane. To isolate the reflections due to the target, we measured the reflection coefficient in the absence of any target, for each frequency and separation distance. We then subtracted this "background" reflection coefficient from the reflection coefficient with the target present. Our results are for the magnitude of this complex difference, which is the part of the reflection coefficient due to the target. The background subtraction is critical, because the background is more than an order of magnitude larger than the target contribution.

\section{MEASURMENT RESULTS}

Figure 2 shows results for a representative frequency $(22 \mathrm{GHz})$ at the range of separation distances. The distances in the graphs refer to cart position; the actual separation distance from antenna to target is obtained by adding an additional $36.5 \mathrm{~cm}$. Figure 2(a) plots the magnitude of the uncorrected reflection coefficient with the target absent (background) and with the larger target present (background plus target), and Fig. 2(b) plots the corrected target reflection coefficient (magnitude of the complex difference between targetpresent and target-absent). Note the different scales in Figs. 2(a) and 2(b). The corrected reflection coefficient lies near or below the VNA manufacturer's quoted uncertainty for these VNA measurements, which is \pm 0.0017 . Thus, the results for the corrected reflection coefficient are consistent with zero over much of the range.

Far more data were collected than can reasonably be presented here. Some representative results are shown in Figs. 3 and 4 . Figure 3 compares the reflectivity of the larger target for the ambienttemperature and heated cases. Any differences 


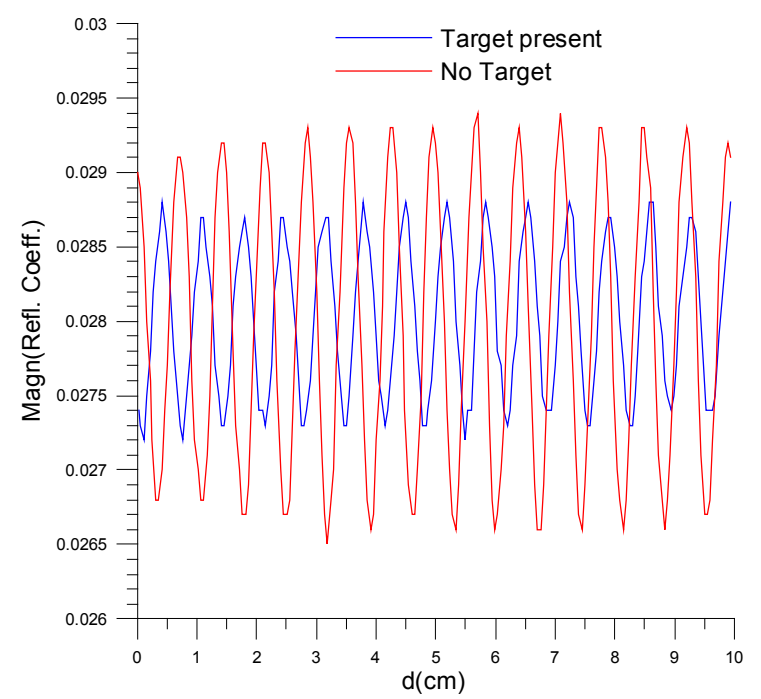

(a)

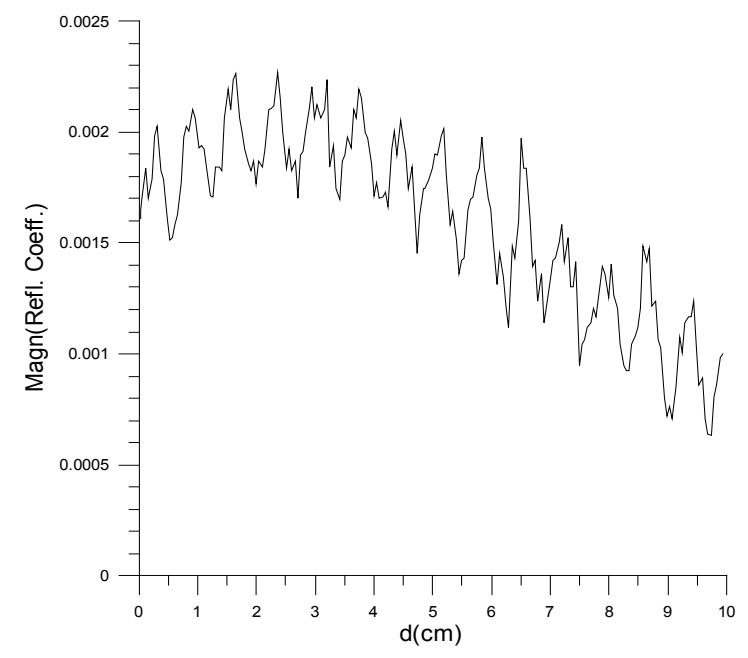

(b)

Figure 2(a). Comparison of reflection coefficient with target present and with no target; 2(b). Corrected target reflection coefficient.

between the two are well below the experimental uncertainty. In addition, the other two targets, not shown here, also showed consistently good agreement between measurements at ambient temperature and hot temperature, indicating a constant reflectivity at variable temperatures. Figure 4 shows the corrected reflection coefficient from a circular, flat aluminum plate of the same diameter as the larger calibration target. Measurements on the aluminum plate were performed for comparison purposes, and also as exploratory tests on the feasibility of using a reflective metal target as a calibration target for reflectivity

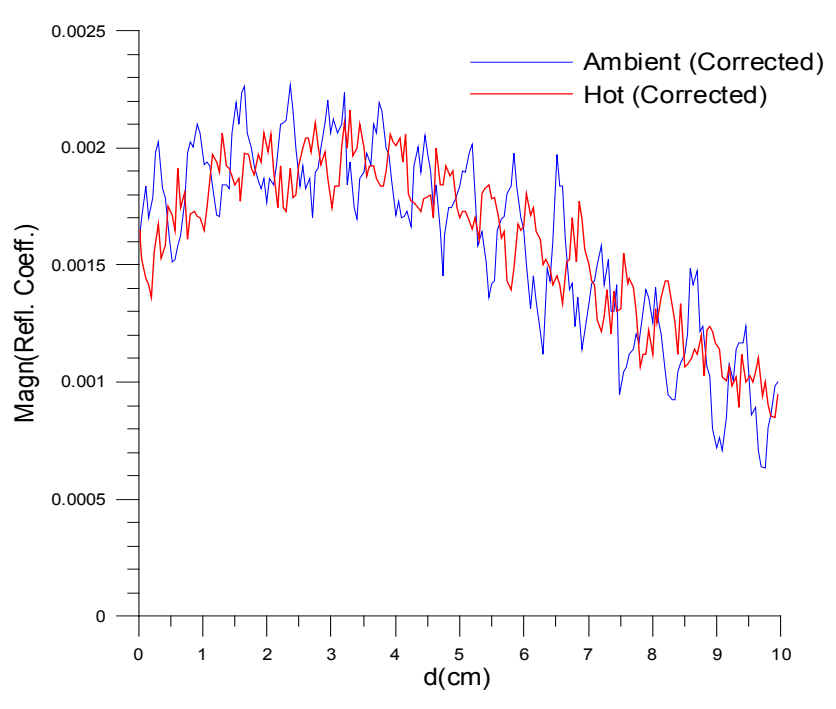

Figure 3. Comparison of hot and ambient targets.

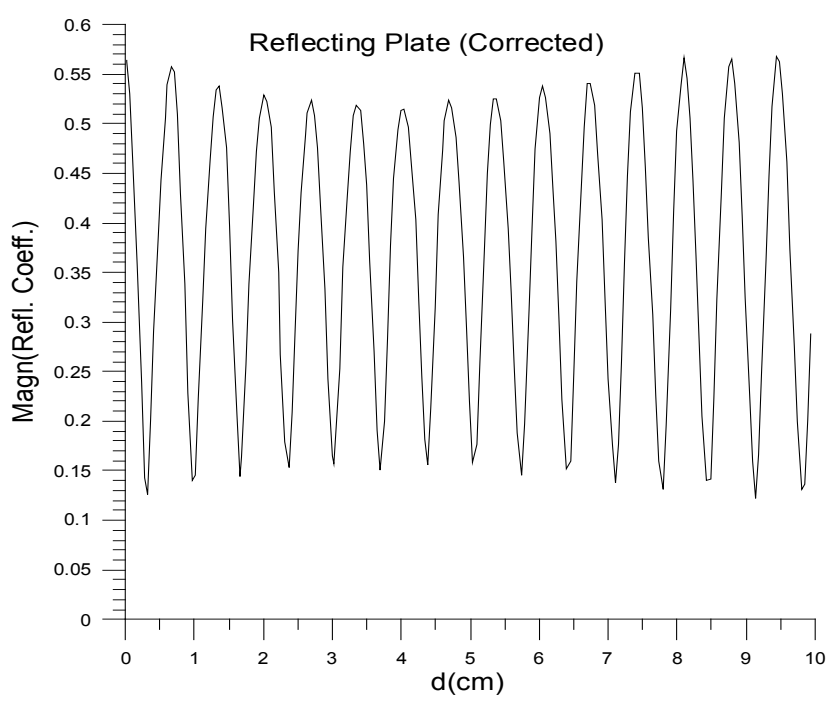

Figure 4. Results for aluminum plate.

measurements. Not surprisingly, the results are two orders of magnitude larger than for the blackbody targets.

\section{DISCUSSION AND FUTURE WORK}

The measured results of the calibration targets suggest close to ideal blackbody characteristics in the measured frequency range. However, a more rigorous study is required to correct some errors that mask the true reflection coefficients of the targets. The descending average of the ripples in the Figure 2 and 3 represents the complexity in the near field, i.e., the reduced effective antenna radiation on the targets 
along the longitudinal direction and the multiple reflection effects between the antenna and the target [4]. Furthermore, the depicted ripple type of response is due to the standing wave effects arising from the mismatch between the horn antenna and the objects under test. Although the ripple amplitude of the targets is much smaller than that of the metal plate, the discernible ripple size measured by the VNA indicates the finite reflection from the target. As it is one of the intrinsic properties of the blackbody target, the reflection coefficient should remain constant, independent of distance. To remove these error terms, we need to use free-space calibration techniques similar to the calibration methods used to treat the guided wave. Future work will be focused on developing de-embedding techniques to calibrate the error boxes present between the antenna waveguide flange and the object under test.

\section{REFERENCES}

[1] J. Randa, A.E. Cox, and D.K. Walker, "Proposal for development of a national microwave brightnesstemperature standard," Proc. SPIE, Vol. 6301, 630105, Sept. 2006.

[2] D.K. Walker, A.E. Cox, J. Randa, K. MacReynolds, R.L. Billinger, and D. Houtz, "Comparison of microwave blackbody target radiometric measurements," this conference, July 2010.

[3] C.Y. Chen, F. Li, Y.J. Yang, and Y.M. Chen, "Emissivity measurement study on wide aperture microwave radiator," ICMMT2008 Proceedings, Beijing 2008.

[4] J. Randa, D.K. Walker, A.E. Cox, and R.L. Billinger, "Errors resulting from the reflectivity of calibration targets," IEEE Trans. Geosci. Remote Sens., vol. 43, no. 1, pp. 50 582005.

[5] D.A. Hill, M. Kanda, E.B. Larsen, G.H. Koepke, and R.D. Orr, "Generating standard reference electromagnetic fields in the NIST anechoic chamber, 0.2 to $40 \mathrm{GHz}$," NIST Technical Note TN1335, March 1990. 